\title{
Uncertainty modeling of random and systematic errors by means of Monte Carlo and fuzzy techniques
}

\author{
Hamza Alkhatib, Ingo Neumann and Hansjörg Kutterer
}

\begin{abstract}
The standard reference in uncertainty modeling is the "Guide to the Expression of Uncertainty in Measurement (GUM)". GUM groups the occurring uncertain quantities into "Type A" and "Type B". Uncertainties of "Type A" are determined with the classical statistical methods, while "Type B" is subject to other uncertainties which are obtained by experience and knowledge about an instrument or a measurement process. Both types of uncertainty can have random and systematic error components. Our study focuses on a detailed comparison of probability and fuzzy-random approaches for handling and propagating the different uncertainties, especially those of "Type B". Whereas a probabilistic approach treats all uncertainties as having a random nature, the fuzzy technique distinguishes between random and deterministic errors. In the fuzzy-random approach the random components are modeled in a stochastic framework, and the deterministic uncertainties are treated by means of a range-of-values search problem. The applied procedure is outlined showing both the theory and a numerical example for the evaluation of uncertainties in an application for terrestrial laserscanning (TLS).
\end{abstract}

Keywords. GUM, Monte Carlo methods, fuzzy methods.

\section{Introduction}

The "Guide to the Expression of Uncertainty in Measurement (GUM)" is a standard reference in uncertainty modeling in engineering and mathematical sciences, cf. ISO (1995). GUM groups the occurring uncertain quantities into "Type A" and "Type B". Uncertainties of "Type A" are determined with the classical statistical methods, while "Type B" is subject to other uncertainties which are obtained by experience and knowledge about an instrument or a measurement process. Whereas the uncertainties of the quantities of "Type A" can be estimated based on repeated measurement of the quantity of interest, the estimated uncertainties of the quantities of "Type B" are based on expert knowledge, e.g., the technical knowledge about an instrumental error source. Both types of uncertainty can have random and systematic error components:

- A random error $\varepsilon$ arises from non predictable variations of some influence factors under seemingly the same actual conditions (non reproducible effects), see, e.g., Bandemer (2006, pp. 63 ff).
- A systematic error $\delta$ is due to non controllable effects during the measurement and the preprocessing steps of the measurement, it biases the output quantity $y$. Although systematic errors are unknown, they bias the measurement result in one direction (reproducible, but unknown effects), see, e.g., Grabe (2005).

GUM defines an output quantity $y$ as a function of input quantities $\mathbf{z}$. The input quantities can be considered as influence parameters which, e.g., can be relevant in pre-processing steps:

$$
y=f\left(z_{1}, z_{2}, \ldots, z_{n}\right)=f(\mathbf{z})
$$

with $f(\cdot)$ the observation model and $n$ the number of input quantities $\mathbf{z}$ :

- “..., whose values and uncertainties are directly determined in the current measurement (original measurement)."

- “..., whose values and uncertainties are brought into the measurement from external sources, like the values from a calibration for an instrument (influence factor) (ISO 1995, Chapter 4.1.3)."

Please note that in general the input quantities $z_{i}$ may be a measurement result $y$ themselves. In order to have a clear representation, only the case where $z_{i}$ is a measurement or an influence factor is treated in this paper. The quantity $z_{i}$ can be carrier of both random and systematic errors. GUM proposes to treat random and systematic errors in a stochastic framework. It introduces variances to describe their uncertainties. Let us denote the function $f(\cdot)$ from equation (1) as observation model and divide the uncertain influence factors into three groups: additional information, sensor parameters, and model constants. Whereas the uncertainty of the original measurement is usually of "Type A", the uncertainty of the influence factors can be of "Type A" or "Type B". Figure 1 shows the interaction between the measurement, the influence factors and the observation model. Systematic errors of the input quantities are meaningful by many reasons:

- The model constants are only partially representative for the given situation (e.g., the model constants for the refraction index for distance measurements).

- The number of additional information (measurements) may be too small to estimate reliable distributions for a random treatment.

- Measurement results are affected by rounding errors. 


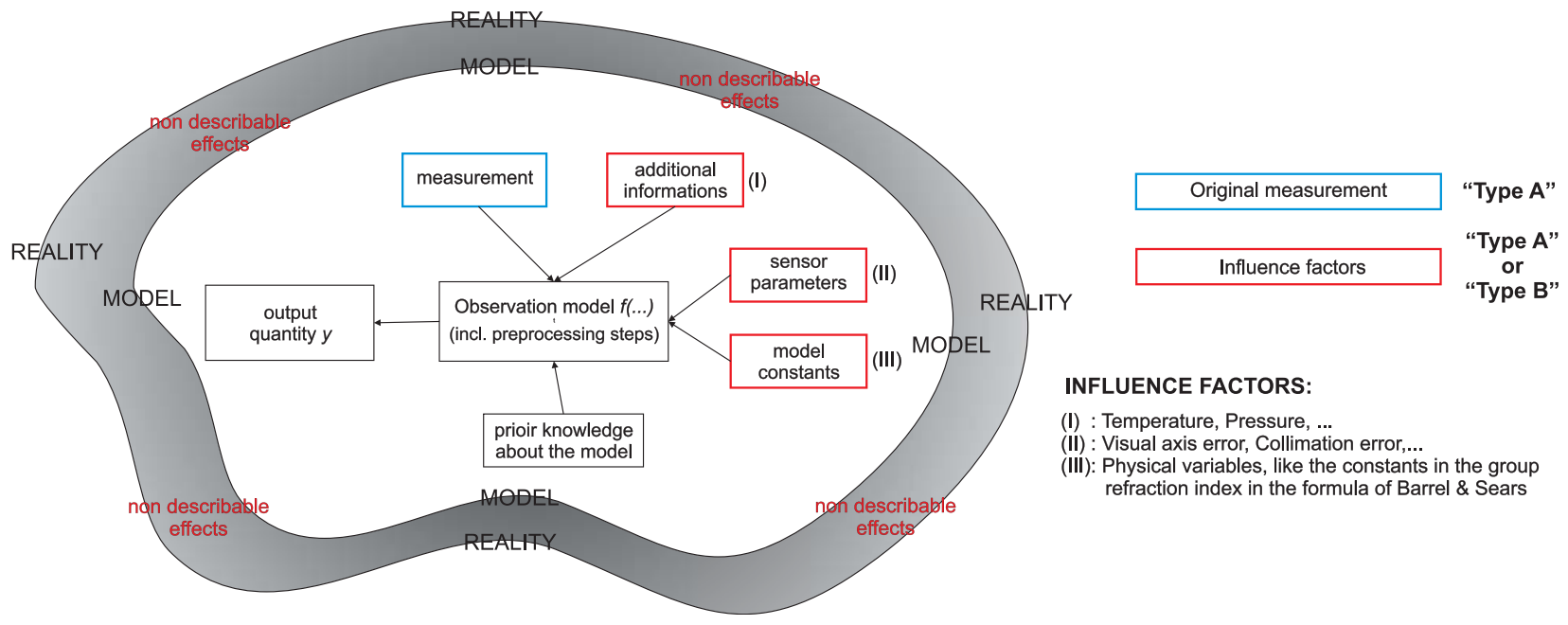

Figure 1: Interaction between input quantities, the observation model and the output quantities.

The GUM, cf. ISO (1995, p. 19), describes an approach for determining the standard uncertainty $u(y)$ of the measurement result $y$ from the standard uncertainties and correlation coefficients associated with the input estimates using the law of propagation of uncertainties (LOP):

$$
\begin{aligned}
u^{2}(y)= & \sum_{i=1}^{n} c_{i}^{2} u^{2}\left(z_{i}\right) \\
& +2 \sum_{i=1}^{n-1} \sum_{j=i+1}^{n} c_{i} c_{j} u\left(z_{i}\right) u\left(z_{j}\right) r\left(z_{i}, z_{j}\right),
\end{aligned}
$$

where the quantities $c_{1}, c_{2}, \ldots, c_{n}$ are partial derivatives of $y=f(\mathbf{z})$ with respect to the random inputs $Z_{1}, Z_{2}, \ldots, Z_{n}$ evaluated at the realization $z_{1}, z_{2}, \ldots, z_{n}$ respectively, and $r\left(z_{i}, z_{j}\right)$ is the correlation coefficient between $Z_{i}$ and $Z_{j}$ for $i, j=1, \ldots, n$ and $i \neq j$. The equation (2) delivers the combined standard uncertainty $u(y)$ of the measurement result $y$ from the standard uncertainties and correlation coefficients associated with the input estimates using the LOP of uncertainties.

Equation (2) can be reformulated in matrix notation, cf., e.g., Koch (1999, p. 100):

$$
u^{2}(y)=\mathbf{A} \Sigma_{z z} \mathbf{A}^{T},
$$

where the matrix $\mathbf{A}$ contains the partial derivatives $y=f(\mathbf{z})$ with respect to $Z_{1}, Z_{2}, \ldots, Z_{n}$, that is $\mathbf{A}=$ $\left[\frac{\partial f}{\partial z_{1}}, \ldots, \frac{\partial f}{\partial z_{n}}\right]$, and $\Sigma_{z z}$ is the uncertainty matrix of the input quantities $\mathbf{z}$.

Unfortunately, in many practical applications, the models are neither linear nor can be approximated by a linearized model using Taylor series expansions. As a consequence of the high non-linearity, the LOP could be difficult to use Hennes (2007). For cases where the model for evaluating the uncertainty is strongly nonlinear or highly complicated to linearize (using Taylor series expansions), the GUM framework will not be satisfied. That is because the standard GUM framework uses only a measurement model linearized about the best available estimates of the input quantities. For this reason the Extension of GUM (ISO 2007) recommends the propagation of uncertainties using a probabilistic approach. Within the mentioned approach the propagation of uncertainties is numerically treated by Monte Carlo (MC) techniques. The difference between the GUM (ISO 1995) and the extension of GUM (ISO 2007) (the so called probabilistic approach) in case of nonlinearity and/or Non-Gaussianity will be not significantly differ in the first and the second central moments but rather in the estimate of the confidence region, which are reflected in the non-Gaussian PDF of the output quantities.

The acceptance of MC techniques has significantly increased during the last decade. Consequently, it's widely used within many scientific disciplines. Hennes (2007) suggested to use MC simulations instead of the treatment of the combined uncertainties by applying the LOP. Siebert and Sommer (2004) recommended a MC based method to evaluate the measurement uncertainties in non-linear models. Koch (2008a) suggested the determination of the uncertainty according to GUM by a Bayesian confidence interval using MC simulation. The approach has been explained in detail and applied to the results of terrestrial laserscanning (TLS). Furthermore, the approach has been extended in Koch (2008b) to evaluate uncertainties of correlated measurements by another application in TLS.

GUM assumes that random and systematic uncertainties are both appropriately handled by means of power density functions (PDFs). Neumann (2009, pp. $24 \mathrm{ff})$ and the references therein show that a "pure" probabilistic approach in some cases can lead to a too optimistic evaluation of uncertainties. Too optimistic evaluation means that, e.g., the confidence intervals of output quantities are too narrow in comparison to the true values. This shall be highlighted with two references. On the one hand McNish (1962) presents in his paper a too optimistic estima- 
tion of confidence intervals for the measurement of the speed of light. On the other hand Stigler (1996) and Youden (1972) illustrate a difference between confidence intervals with the true values for the astronomical unit. Hence, in this paper an alternative approach for evaluating the uncertainties will be introduced. In this approach the systematic component is evaluated using fuzzy techniques (in contrast to the "pure" probabilistic approach). The fuzzy techniques have proven to be an appropriate solution for the description of uncertainties and were applied in different science and engineering applications.

The basic idea in this paper is related to the so-called Fuzzy-Random-Variables (FRV) which are based on a combination of probability theory and fuzzy theory, see Kwakernaak (1978) and Kwakernaak (1979) for more details. For the theory of FRV the reader is referred to Terán (2007). Further discussions of fuzzy techniques in the context of GUM can be also found in Mauris et al. (2001). The description of the systematic uncertainty component of the input quantities with the fuzzy-random approach leads to a more pessimistic uncertainty of the output quantity. In this paper the probabilistic approach and fuzzyrandom approach shall be thoroughly compared to each other from a theoretical point of view and using a practical example in TLS.

The paper is organized as follows: First we will describe the general idea of MC techniques to describe measurement uncertainties in the context of GUM; second a fuzzy-random approach to handle these measurement uncertainties is introduced. Then, the computation steps of both approaches are compared with the GUM approach. In the fifth section all three methods are applied to TLS and the obtained results are critically compared to each other. The paper finishes with a discussion and an outlook for further research.

\section{Uncertainty modeling with probabilistic approach}

In MC techniques, both the random and the systematic components of the uncertainty are treated as having a random nature. Please note that not the systematic component itself is modeled as random, it is the knowledge about the systematic component for which a PDF is introduced (Koch 2007). The GUM suggested in some cases to select the PDF of the input quantities as rectangular, triangular, and trapezoidal (ISO 1995). In these cases, it is hard or even impossible to obtain the estimate of the uncertainty for the output quantity in a closed mathematical form. An alternative to modeling and propagating first and second moments according to equation (2) or (3) is propagating PDFs of the observation model from equation (1) by MC simulations:

$$
Y=f\left(Z_{1}, Z_{2}, \ldots, Z_{n}\right)=f(\mathbf{Z}) .
$$

Here $Y$ represents a random output quantity and $Z_{1}, Z_{2}, \ldots, Z_{n}$ are the $n$ random inputs.

\subsection{Sampling from PDF}

Any MC simulation requires random numbers. Random numbers are generated on a computer by means of deterministic procedures. Mostly, rectangular distributed random numbers are generated, which may then in turn be transformed into random numbers of random variables having other PDFs (Gentle 2003).

Starting from pseudo-random numbers $u_{1}, u_{2}, \ldots, u_{n}$, generated by one of the standard methods such as linear congruence method (Koch 2007, p. 183), some random numbers $x_{1}, x_{2}, \ldots, x_{n}$ may be generated which may be viewed as realizations of random variables $X_{1}, X_{2}, \ldots, X_{n}$ with another PDF, for instance as realizations $x_{1}, x_{2}, \ldots, x_{n}$ of normally distributed random variables. This process makes in particular use of the so-called inversion method or acceptancerejection method. A far more comprehensive discussion of such algorithms can be found, e.g., in Gentle (2003) or Koch (2007). Multivariate uniform, trapezoidal and triangular PDFs might be needed for evaluating the uncertainties of the measurements according to GUM. In this paper, the inversion method is used to generate rectangular-distributed and triangular-distributed random numbers. The main reason for the selection of the inversion method is its implementation simplicity. Detailed explanation of this method can be found, e.g., in Gentle (2003).

To demonstrate the modeling of uncertainties with a MC simulation in Section 5, the generation algorithms of random numbers from rectangular, triangular and normal PDF will be shortly described according to Koch (2007):

- Generation of rectangular-distributed random numbers: In a rectangular PDF, the values of a random variable lie within an interval $\left[a_{-}, a_{+}\right]$, where $a_{-}$ and $a_{+}$are the distribution parameters, the upper and the lower limits of the rectangular PDF. That means, there is no specific knowledge about the values within the interval, cf. Koch (2008a) (typical examples are error bounds, rounding errors and digitalization errors). The PDF for a continuous random variable of the uniform PDF, can be defined by (cf., e.g., Koch (2007, p. 20)):

$p\left(x \mid a_{-}, a_{+}\right)= \begin{cases}\frac{1}{a_{+}-a_{-}} & \text {if } a_{-} \leq x \leq a_{+}, \\ 0 & \text { if } x<a_{-} \text {and } x>a_{+} .\end{cases}$

The expected value $E(X)$ and the variance $V(X)$ of the rectangular PDF are given by (cf. Koch (2008a)):

$$
E(X)=\frac{a_{+}+a_{-}}{2}, \quad V(X)=\frac{\left(a_{+}-a_{-}\right)^{2}}{12} .
$$

- Generation of triangular-distributed random numbers: The symmetric triangular PDF is a special 
case of the symmetric trapezoidal PDF. As an illustrative example for this type of distributions, the triangular PDF is suggested as a distribution for an addition constant of an instrument. The symmetric triangular cumulative distribution function $(\mathrm{CDF})$ is then given by:

$F\left(x \mid a_{-}, a_{+}\right)= \begin{cases}\frac{\left(x-a_{-}\right)^{2}}{2 a^{2}} & \text { if } a_{-} \leq x \leq \frac{a_{+}+a_{-}}{2}, \\ 1-\frac{\left(a_{+}-x\right)^{2}}{2 a^{2}} & \text { if } \frac{a_{+}+a_{-}}{2} \leq x \leq a_{+},\end{cases}$

where $a=\frac{a_{+}-a_{-}}{2}$. In order to generate random numbers $x_{1}, x_{2}, \ldots, x_{n}$ from the triangular distribution, the inverse function of the CDF in equation (7) is derived. The method is similar to the one used to generate random variables from the uniform distribution. One starts with one-dimensional vector of standard uniform-distributed random numbers, $H \sim \mathscr{U}(0,1)$. These can be transformed to the desired distribution using the inverse function of the $\mathrm{CDF}(7)$ :

$F^{-1}(h)= \begin{cases}a_{-}+\sqrt{2 h a^{2}} & \text { if } 0 \leq h \leq \frac{1}{2}, \\ a_{+}-\sqrt{2 a^{2}(1-h)} & \text { if } 0 \leq(1-h) \leq \frac{1}{2} .\end{cases}$

The expected value and the variance of the triangular PDF are given by (cf. Koch (2008a)):

$$
E(X)=\frac{a_{+}+a_{-}}{2}, \quad V(X)=\frac{\left(a_{+}-a_{-}\right)^{2}}{24} .
$$

- Generation of correlated normally-distributed random numbers: The normal PDF is the most widely used PDF in statistics. The one-dimensional normal PDF is fully described by its expected value $\mu$ and its variance $\sigma^{2}$, that is $X \sim \mathscr{N}\left(\mu, \sigma^{2}\right)$. The multinormal PDF is a building block for the some input quantities of the random variables in equation (4). It is well known that the multinormal PDF is fully characterized by its expected value $\boldsymbol{\mu} \in \mathbb{R}^{n}$ and its variance-covariance matrix $\Sigma \in \mathbb{R}^{n \times n}$, where $n$ is the length of the random vector. Consider a vector $\mathbf{Z}=\left(Z_{1}, \ldots, Z_{n}\right)^{T}$ of independent standard-normally distributed random variables $\mathbf{Z} \sim \mathscr{N}(\mathbf{0}, \mathbf{I})$. As $\Sigma$ is positive definite then there exists the Cholesky decomposition $\Sigma=\mathbf{R}^{T} \mathbf{R}$, where $\mathbf{R}$ is an $n \times n$ upper triangular matrix. The standard normal numbers can be then transformed to the desired distribution (that is $\mathbf{X} \sim \mathscr{N}(\boldsymbol{\mu}, \boldsymbol{\Sigma})$ ) using $\mathbf{X}=\mathbf{R}^{T} \mathbf{Z}+\boldsymbol{\mu}$. Another possibility to generate multivariate normal random variables is to factorize the covariance matrix $\Sigma$ using singular value decomposition, cf., e.g., Koch (2007, p. 196).

\subsection{MC technique to evaluate uncertainty}

The MC techniques are of great importance for uncertainty evaluation. With a set of generated samples the PDF for the value of the output quantity $Y$ in equation (4) will be numerically approximated. MC approaches to estimate the uncertainty include the following steps:
- Step 1: A set of random samples $z_{1}, z_{2}, \ldots, z_{n}$, which have the size $n$, is generated from the PDF for each random input quantity $Z_{1}, Z_{2}, \ldots, Z_{n}$. The sampling procedure is repeated $M$ times for every input quantity.

- Step 2: The output quantities $y$ will be then calculated by:

$$
y^{(i)}=f\left(z_{1}^{(i)}, z_{2}^{(i)}, \ldots, z_{n}^{(i)}\right)=f\left(\mathbf{z}^{(i)}\right),
$$

where $i=1, \ldots, M$ are the generated samples of the random output quantity $Y$.

- Step 3: Particularly relevant estimates of any statistical quantities can be calculated:

(1) the expectation of the output quantity

$$
\hat{E}(f(\mathbf{z}))=\hat{E}(y)=\frac{1}{M} \sum_{i=1}^{M} f\left(\mathbf{z}^{(i)}\right)
$$

(2) the estimate of the variance of the output quantity (Alkhatib 2007)

$$
\begin{aligned}
\hat{\mathbf{D}}(y)= & \frac{1}{M} \sum_{i=1}^{M}\left(f\left(\mathbf{z}^{(i)}\right)-\hat{E}(f(\mathbf{z}))\right) \\
& \cdot\left(f\left(\mathbf{z}^{(i)}\right)-\hat{E}(f(\mathbf{z}))\right)^{T} .
\end{aligned}
$$

\subsection{Confidence intervals in the MC case}

To compute the confidence interval by MC simulation of the output quantity with the significance level of $\gamma$ (it may be denoted as $y_{\mathrm{conf}, \mathrm{MC}}=[y, \bar{y}]$ ), one has to order the independent variates $y^{(i)}$ from the smallest to the largest. An numerically computed $100(1-2 \gamma) \%$ confidence interval for the random variable $Y$ is then given (cf. Buckland (1984)):

$$
y_{\text {conf }, \mathrm{MC}}=\left[\underline{y}=y_{j}, \bar{y}=y_{k}\right],
$$

where $j=(M+1) \gamma$ and $k=(M+1)(1-\gamma)$. The resulting $j$ and $k$ are in general not integer numbers. In order to round to the nearest integer the linear interpolation between as example $y_{i}$ and $y_{i+1}$, where $i<j<i+1$ is used. Another way to compute the MC confidence region is described in Koch (2008a).

\section{A fuzzy-random approach to uncertainty modeling}

In this section, a fuzzy-random approach to uncertainty modeling in the context of GUM is introduced. Fuzzy techniques have proven to be an appropriate solution for the description of uncertainties. Recently, many procedures have been introduced in different engineering applications, cf., e.g., Fellin et al. (2005), Kreinovich et al. (2006), Möller and Beer (2004). This includes discussions about combined approaches in fuzzy theory, interval mathematics and probability theory (Ferson et al. 2002) and linguistic motivations, see, e.g., Kreinovich (2007).

In the fuzzy-random approach we distinguish between random and systematic errors in the propagation process of the uncertainties of the input quanti- 
ties to the output quantity $y$. Whereas the random part is treated with the LOP or with the MC technique, systematic errors are propagated by means of a range-of-values search problem (see Section 3.2). Both types of uncertainty are modeled in a comprehensive way, using fuzzy intervals (see Section 3.1). This procedure is in full accordance with the recommendations of the GUM. However, the difference is in the treatment of the uncertainty about the systematic errors, for which no variances but interval radii are introduced.

A comprehensive modeling of uncertainties from the observations to the parameters of interest (including statistical tests) with the approach used here is given in Neumann and Kutterer (2006) and Neumann and Kutterer (2009).

\subsection{Uncertainty modeling using fuzzy intervals}

The random and systematic components of the uncertainties are characterized with a special case of fuzzy theory, the so called Fuzzy-Randomness (Möller and Beer 2004, Viertl 1996). Each uncertain quantity $z_{i}$ is exclusively modeled in terms of fuzzy intervals. A fuzzy interval $\tilde{A}$ is uniquely defined by its membership function $m_{\tilde{A}}(x)$ over the set $\mathbb{R}$ of real numbers with a membership degree between 0 and 1:

$$
\begin{aligned}
\tilde{A}:= & \left\{\left(x, m_{\tilde{A}}(x)\right) \mid x \in \mathbb{R}\right\} \\
& \text { with } m_{\tilde{A}}(x): \mathbb{R} \rightarrow[0,1] .
\end{aligned}
$$

The membership function of a fuzzy interval can be described by its left $L(\cdot)$ and right $R(\cdot)$ reference function (see also Figure 2):

$$
m_{\tilde{A}}(x)= \begin{cases}L\left[\frac{X_{m}-x-r}{c_{l}}\right] & \text { for } x<X_{m}-r \\ 1 & \text { for } X_{m}-r \leq x \leq X_{m}+r \\ R\left[\frac{x-X_{m}-r}{c_{r}}\right] & \text { for } x>X_{m}+r\end{cases}
$$

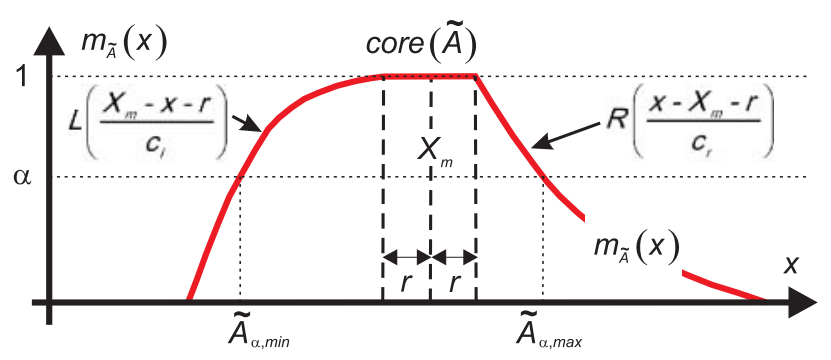

Figure 2: Fuzzy interval and its $\alpha$-cut.

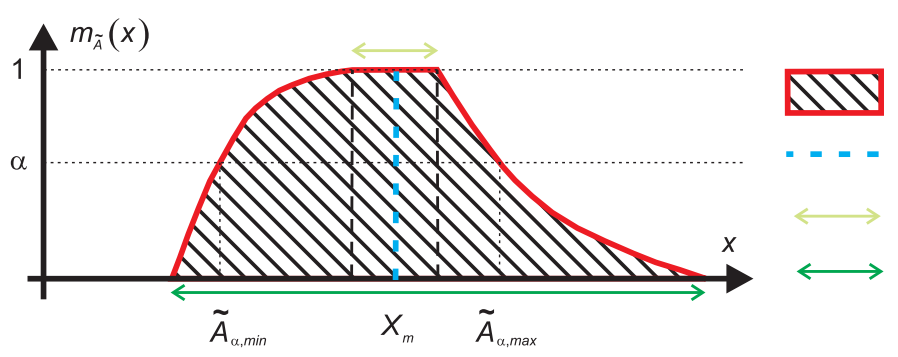

Carrier for the systematic errors

Carrier for the random errors

Optimistic range for the systematic error

Pessimistic range for the systematic error with $X_{m}$ denoting the midpoint, $r$ its radius, and $c_{l}, c_{r}$ the spread parameters of the monotonously decreasing reference functions (convex fuzzy intervals).

The $\alpha$-cut $\tilde{A}_{\alpha}$ with $\alpha \in(0,1]$ of a fuzzy interval $\tilde{A}$ is defined by

$$
\tilde{A}_{\alpha}:=\left\{x \in \mathbb{R} \mid m_{\tilde{A}(x)} \geq \alpha\right\} .
$$

Each $\alpha$-cut represents in case of monotonously decreasing reference functions an interval. The lower $\tilde{A}_{\alpha, \min }$ and the upper bound $\tilde{A}_{\alpha, \max }$ of an $\alpha$-cut and its radius $\tilde{A}_{\alpha, r}$ are

$$
\begin{aligned}
\tilde{A}_{\alpha, \min } & =\min \left(\tilde{A}_{\alpha}\right) \\
\tilde{A}_{\alpha, \max } & =\max \left(\tilde{A}_{\alpha}\right) \\
\tilde{A}_{\alpha, r} & =\frac{\tilde{A}_{\alpha, \max }-\tilde{A}_{\alpha, \min }}{2} .
\end{aligned}
$$

The $\alpha$-cut with $\alpha=1$ is called the core of $\tilde{A}$ (see Figure 2) with $\operatorname{core}(\tilde{A}):=\left\{x \in \mathbb{R} \mid m_{\tilde{A}}(x)=1\right\}$, and the set with a positive membership function is the support of $\tilde{A}$, that is $\operatorname{supp}(\tilde{A}):=\left\{x \in \mathbb{R} \mid m_{\tilde{A}}(x)>0\right\}$. The membership function can also be constructed on the $\alpha$-cuts:

$$
m_{\tilde{A}}(x)=\sup _{\alpha \in(0,1]} \alpha \cdot m_{\tilde{A}_{\alpha}}(x)
$$

In this concept fuzzy intervals serve as basic quantities; their midpoints $X_{m}$ are considered in the following as random variables and their spreads $c_{l}, c_{r}$ describe the range of the uncertainty about systematic errors. If one component has random uncertainty only, then this input quantity only consists of a single midpoint as core with radius $r=0$ and without a left and right reference function. In contrast to the probabilistic approach, the membership function of a fuzzy interval cannot be interpreted in a probabilistic meaning. Therefore the propagation of the systematic uncertainties has to be modified accordingly (see Section 3.2). In the fuzzy case, we model the influence of a systematic component of the uncertainty on the output quantity $y$. Figure 3 shows the interpretation of a fuzzy interval in the here presented approach. The construction of the membership function can be based on expert knowledge. Each expert provides a range-of-values (an interval) for a systematic error which he considers as realistic. The core of a fuzzy interval describes the range-of-values where all experts agree that these values are possible (most

Figure 3: Interpretation of a fuzzy interval. 
optimistic case). The support represents the most pessimistic expert opinion for the range-of-values for the systematic error. The above described procedure to construct fuzzy intervals is based on the theory of nested sets, see Nguyen and Kreinovich (1996) for a detailed explanation and Neumann (2009) in the context of uncertainty propagation in parameter estimation, and in the context of hypothesis tests.

\subsection{Uncertainty propagation with the extension principle}

The propagation process of the random and systematic errors is separated in two parts. Whereas the random components are treated with the LOP (GUM, Chapter 5.2) or with the MC technique (see Section 2 ), the propagation of the systematic errors is a range-of-values search problem. The extension principle introduced by Zadeh (1965) serves as basic rule to propagate both types of uncertainty.

Therefore the mapping $\tau: y=f(\mathbf{z}), \mathbf{z} \in \mathbb{R}^{n} \rightarrow y \in \mathbb{R}$ can be extended to the case of fuzzy intervals $\tilde{\tau}: \mathscr{J}\left(\mathbb{R}^{n}\right) \rightarrow \mathscr{J}(\mathbb{R})$ with $\mathscr{J}\left(\mathbb{R}^{n}\right)$ the fuzzy intervals over the real numbers in $\mathbb{R}^{n}$ and $\mathscr{J}(\mathbb{R})$ the fuzzy intervals in $\mathbb{R}$ (Dubois and Prade 1980, p. 37):

$$
\tilde{y}_{\alpha, \min }=\min _{z_{i} \in\left[\tilde{z}_{z_{\alpha}}\right]} f(\mathbf{z}), \quad \tilde{y}_{\alpha, \max }=\max _{z_{i} \in\left[\tilde{z}_{i_{\alpha}}\right]} f(\mathbf{z}),
$$

with

$$
\tilde{y}_{\alpha}=\left[\tilde{y}_{\alpha, \min }, \tilde{y}_{\alpha, \max }\right], \quad m_{\tilde{y}}(x)=\sup _{\alpha \in(0,1]} \alpha \cdot m_{\tilde{y}_{\alpha}}(x) .
$$

The computation of the membership function for the measurement results is based on the $\alpha$-cuts $\tilde{\mathbf{z}}_{\alpha}$ of the input quantities, within the optimization problem of equation (19). The approximate midpoint of the fuzzy interval for the output quantity $y_{m}$ is

$$
y_{m}=f\left(z_{1_{m}}, z_{2_{m}}, \ldots, z_{n_{m}}\right)=f\left(\mathbf{z}_{m}\right) .
$$

In general, the solution in equation (21) is only correct for sufficiently linear functions $f(\cdot)$. For practical applications linear reference functions for the membership function of the input quantities and a linear function $f(\cdot)$ are of interest. In these cases, the propagation process only needs to be applied for the $\alpha$-cuts with $\alpha=0$ and $\alpha=1$.

\subsection{Confidence intervals in the fuzzy case}

The computation of confidence intervals in the fuzzyrandom approach is also based on the extension principle. Let $k\left(Z_{1}, Z_{2}, \ldots, Z_{n}\right)=k(\mathbf{Z})$ be a confidence function for the output quantity $Y$ and the realizations of the random variables $\mathbf{Z}$ are given by fuzzy intervals $\tilde{z}_{1}, \tilde{z}_{2}, \ldots, \tilde{z}_{n}$. Then the membership function $m_{\tilde{y}_{\text {conf, fuzzy }}}$ of the fuzzy confidence interval $\tilde{y}_{\text {conf, fuzzy }}$ for the output quantity is given by

$$
\begin{aligned}
\tilde{y}_{\text {conf, fuzzy }}= & \tilde{k}\left(\tilde{z}_{1}, \ldots, \tilde{z}_{n}\right)=\tilde{k}(\tilde{\mathbf{z}})=m_{\tilde{y}_{\text {conf fuzzy }}}(y) \\
= & \sup _{\substack{\left(z_{1}, \ldots, z_{n}\right) \in \mathbb{R}_{1} \times \cdots \times \mathbb{R}_{n} \\
y \in k\left(z_{1}, \ldots, z_{n}\right)}} \min \left(m_{\tilde{z}_{1}}\left(z_{1}\right), \ldots, m_{\tilde{z}_{n}}\left(z_{n}\right)\right) \\
& \forall y \in \mathbb{R} .
\end{aligned}
$$

A geometric interpretation of equation (22) for nonlinear functions is difficult. In case of sufficiently linear functions $f\left(\mathbf{z}_{m}\right)$ the fuzzy confidence interval $\tilde{y}_{\text {conf, fuzzy }}$ for the output quantity based on equation (22) is obtained by the combination of both uncertainty components (Kutterer 2002):

$$
\begin{array}{r}
\tilde{y}_{\alpha, \text { conf , fuzzy }}\left[\underline{y}-\tilde{y}_{\alpha, r}, \bar{y}+\tilde{y}_{\alpha, r}\right] \\
\text { with } \tilde{y}_{\alpha, r}=\frac{\tilde{y}_{\alpha, \max }-\tilde{y}_{\alpha, \min }}{2} .
\end{array}
$$

Whereas the $\alpha$-level of zero corresponds to the pessimistic case, the optimistic case is obtained for $\alpha=1$. Please note that only the random uncertainty components from the input quantities $\mathbf{z}$ contribute to the lower and upper bound of the MC confidence interval $\tilde{y}_{\text {conf }, \mathrm{MC}}=[y, \bar{y}]$.

\section{Compilation of the GUM, probabilistic, and fuzzy-random approach}

In this section a short compilation of the calculation steps in the GUM, probabilistic, and fuzzy-random approach is given. Figure 4 shows a diagram with the treatment of random and systematic errors of each technique.

In GUM (the left column of Figure 4) the output quantity $Y$ (cf. equation (4)) is treated as a random variable with a specified PDF. The probability function is assumed (or approximated) to be the normal (Gaussian) PDF. The probabilistic approach of $y$ is based on propagating PDFs by numerical MC simulation of the observation model (4) (the middle column of Figure 4). Only if the assumption is fulfilled that the PDFs for all random input quantities $X_{1}, \ldots, X_{n}$ are full specified, a PDF of the output quantity $Y$ can be computed numerically. On the basis of the generated PDF, the expected value $E(y)$ and its uncertainty $u_{\mathrm{MC}}(y)$ can be determined. The systematic error as well as the random error are fully described in the probabilistic approach by means of PDFs/CDFs. In contrast to the probabilistic approach, the fuzzy-random approach distinguishes between random and systematic errors within the uncertainty propagation process. Whereas the random component is treated as random variable with, e.g., the MC simulations, the systematic component is propagated as a range-of-value search problem. The two different propagation principles of random and systematic errors in the fuzzy-random approach can be covered with the so called FRV from Section 3. 


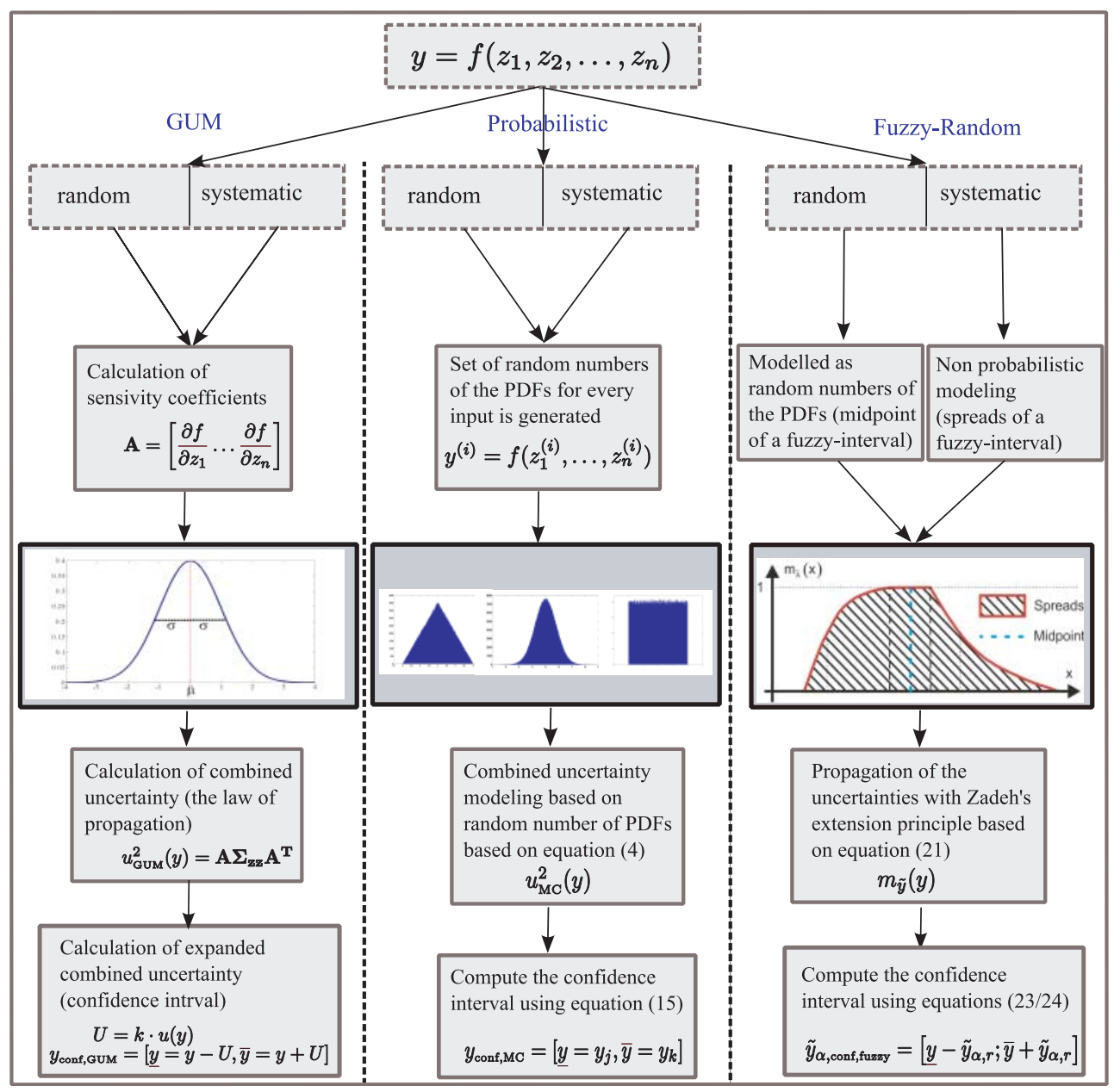

Figure 4: Comparison of the GUM, the probabilistic, and the fuzzy-random approach.

This leads to the uncertainty propagation given in equation (19).

\section{Numerical example for an application to TLS}

In this section a short numerical example for the comparison of the two approaches from Section 2 and 3 is presented. The aim was to detect the vertical displacements of the bridge under load, e.g., due to traffic or train crossings (Strübing 2007). For this reason, a laserscanner of type Leica HDS 4500 was placed beneath the bridge; the measurements in the "Profiler Mode" span the green plane in Figure 5. The discrepancies to the standard case of normal distributed measurements are meaningful by many reasons (see also Section 1). The laserscanner carries out very fast measurements and the measurements are influenced by vibrations due to the traffic load of the bridge. The time series of the vertical height $h_{t}$ of the bridge at the stations $7.28 \mathrm{~m}$ and $21.90 \mathrm{~m}$ can be expressed in the local coordinate system of the laserscanner by the following equation:

$$
h_{t}=s_{t} \cdot \cos \left(\zeta_{t}\right)
$$

with the slope distance $s_{t}$ and the zenith angle $\zeta_{t}$, measured by the laserscanner. The number of measured epochs $q$ is 100 . The vertical displacements $w_{t}$ of the bridge are obtained by subtracting the mean height of the bridge from the time series in equation (24):

$$
w_{t}=h_{t}-\frac{1}{q} \sum_{t=1}^{q} h_{t}=s_{t} \cdot \cos \left(\zeta_{t}\right)-\frac{1}{q} \sum_{t=1}^{q} s_{t} \cdot \cos \left(\zeta_{t}\right) .
$$

The equation (25) is evaluated for two points. The first point 1831 is at station $7.28 \mathrm{~m}$ and point 8987 at station $21.90 \mathrm{~m}$ (see Figure 5).

\subsection{Uncertainties for the measurements and influence factors}

The uncertainty of output quantity $y \triangleq w_{t}$ depends on the following input quantities $z_{i}$ :

- accuracy of the distance $\left(z_{1}\right.$, Type A), and its additional constant $\left(z_{2}\right.$, Type B),

- distance depending term for the accuracy of the distance measurement $\left(z_{3}\right.$, Type B),

- spatial direction of incidence angle of the measured distance under the bridge with respect to the object surface $\left(z_{4}\right.$, Type B), 


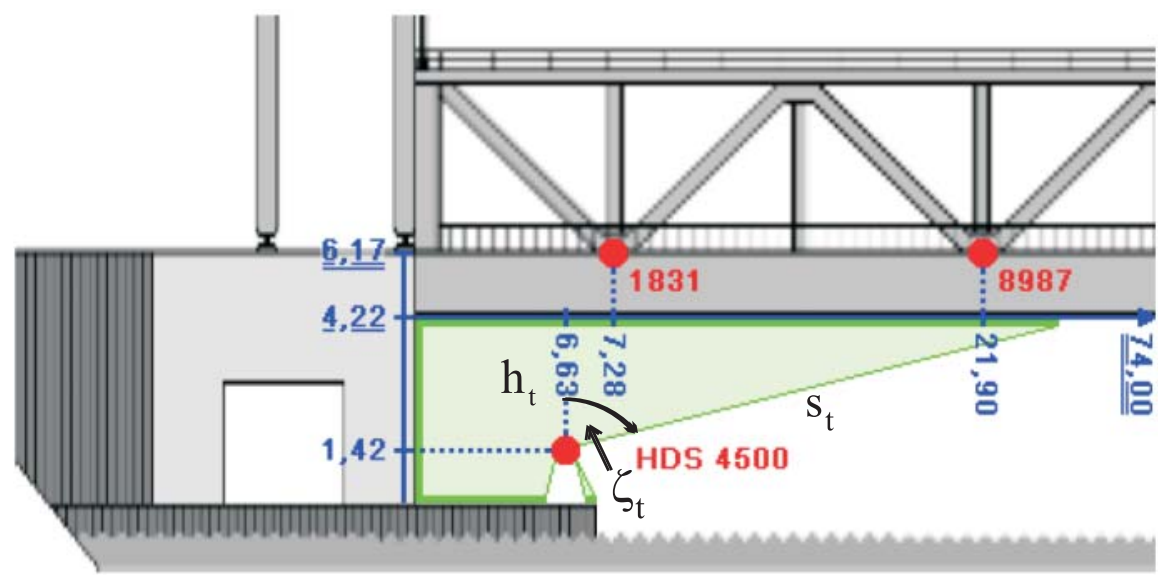

Figure 5: Position of the laserscanner beneath the bridge (Strübing 2007).

Table 1: Uncertainties for the input quantities $\mathbf{z}$.

\begin{tabular}{|c|c|c|c|c|c|}
\hline Input quantity & Error component & $\mathrm{PDF} / m_{\tilde{y}}\left(z_{i}\right)$ & Type & Uncertainty & $u\left(z_{i}\right)$ for GUM \\
\hline$z_{1}$ & random & normal & A & $\sigma=3 \mathrm{~mm}$ & $u\left(z_{1}\right)=3 \mathrm{~mm}$ \\
\hline$z_{2}$ & systematic & triangular & $\mathrm{B}$ & $\begin{array}{l}\left(a_{+}-a_{-}\right) / 2=3 \mathrm{~mm} \\
\tilde{z}_{\alpha=0, r}=3 \mathrm{~mm}\end{array}$ & $u\left(z_{2}\right)=1.225 \mathrm{~mm}$ \\
\hline$z_{3}$ & random & normal & B & $\begin{array}{l}\sigma=0.2 \mathrm{~mm}(1831) \\
\sigma=0.9 \mathrm{~mm}(8987)\end{array}$ & $\begin{array}{l}u\left(z_{3}\right)=0.2 \mathrm{~mm} \quad(1831) \\
u\left(z_{3}\right)=0.9 \mathrm{~mm}(8987)\end{array}$ \\
\hline$z_{4}$ & random & normal & B & $\begin{array}{l}\sigma=2.6 \mathrm{~mm}(1831) \\
\sigma=7.2 \mathrm{~mm}(8987)\end{array}$ & $\begin{array}{l}u\left(z_{4}\right)=2.6 \mathrm{~mm}(1831) \\
u\left(z_{4}\right)=7.2 \mathrm{~mm}(8987)\end{array}$ \\
\hline$z_{5}$ & random & normal & A & $\sigma=20$ mgon & $u\left(z_{5}\right)=20$ mgon \\
\hline$z_{6}$ & systematic & triangular & B & $\begin{array}{l}\left(a_{+}-a_{-}\right) / 2=20 \text { mgon } \\
\tilde{z}_{\alpha=0, r}=20 \text { mgon }\end{array}$ & $u\left(z_{6}\right)=8.165$ mgon \\
\hline$z_{7}$ & systematic & rectangular & B & $\begin{array}{l}\left(a_{+}-a_{-}\right) / 2=10 \text { mgon } \\
\tilde{z}_{\alpha=0, r}=10 \text { mgon }\end{array}$ & $u\left(z_{7}\right)=5.774$ mgon \\
\hline
\end{tabular}

- accuracy of the zenith angle $\left(z_{5}\right.$, Type A) and the vertical index error $\left(z_{6}\right.$, Type B),

- vertical resolution for the zenith angle (the step width of the motor) ( $z_{7}$, Type B).

The uncertainties described by the PDF/membership function for the input quantities $z_{i}$ are given in Table 1. The assumptions for the uncertainties of $z_{1}, z_{5}$, and $z_{6}$ are based on the technical data from the manufacturer and for the uncertainties of $z_{2}, z_{3}$, and $z_{4}$ on Schulz and Ingensand (2004) and for $z_{7}$ on Reshetyuk (2006). The input quantities $z_{3}$ and $z_{4}$ have a correlation of 0.5 , according to Koch (2008a). The numbers 8987 and 1831 in the brackets represent the point number (see Figure 5). In order to have an easier representation, each input quantity is modeled either as random or as systematic. In general the uncertainty budget of each input quantity may consist of both a random and systematic component. Please note that the uncertainties of the input quantities of $z_{2}, z_{6}$, and $z_{7}$ are fully described with their distribution parameters (in this case the upper limit $a_{+}$and the lower limit $\left.a_{-}\right)$.

\subsection{Specification and discussion of the numerical results}

This study focuses on the comparison of the three different techniques to model and propagate the occurring uncertainties in Table 1. The PDFs and the order of magnitude of the uncertainties from Table 1 are in our opinion realistic regarding typical situations in the applications. Their description must be carefully examined in future work, but this is not part of the paper. First the LOP-technique (according to GUM) is used to propagate the uncertainties (Section 5.2.1). Then the MC- and the fuzzy technique from Sections 2 and 3 were applied.

\subsubsection{Uncertainties obtained by the GUM approach}

The propagation process of the uncertainties in the GUM approach is separated into two steps. In the first step, the combined uncertainty

$$
u_{h_{t}}^{2}=\mathbf{A} \Sigma_{\mathbf{z z}} \mathbf{A}^{T},
$$

for the vertical height $h_{t}$ of the bridge is computed. 
The matrices from equation (3) are given by

$$
\begin{aligned}
& \mathbf{A}=\left[\begin{array}{c}
\cos \zeta_{t} \\
\cos \zeta_{t} \\
\cos \zeta_{t} \\
\cos \zeta_{t} \\
-s_{t} \sin \zeta_{t} \\
-s_{t} \sin \zeta_{t} \\
-S_{t} \sin \zeta_{t}
\end{array}\right], \\
& \Sigma_{\mathbf{z z}}=\left[\begin{array}{ccccccc}
u^{2}\left(z_{1}\right) & 0 & 0 & 0 & 0 & 0 & 0 \\
0 & u^{2}\left(z_{2}\right) & 0 & 0 & 0 & 0 & 0 \\
0 & 0 & u^{2}\left(z_{3}\right) & 0.5 u\left(z_{3}\right) u\left(z_{4}\right) & 0 & 0 & 0 \\
0 & 0 & 0.5 u\left(z_{4}\right) u\left(z_{3}\right) & u^{2}\left(z_{4}\right) & 0 & 0 & 0 \\
0 & 0 & 0 & 0 & u^{2}\left(z_{5}\right) & 0 & 0 \\
0 & 0 & 0 & 0 & 0 & u^{2}\left(z_{6}\right) & 0 \\
0 & 0 & 0 & 0 & 0 & 0 & u^{2}\left(z_{7}\right)
\end{array}\right] .
\end{aligned}
$$

For an easy presentation we assume that the heights of different epochs $t$ are uncorrelated and their uncertainties are assumed to be equal. Using the LOP from equation (26) for equation (25) then leads to:

$$
\begin{aligned}
u_{w_{t}}^{2} & =\left(\frac{q-1}{q}\right)^{2} u_{h_{t}}^{2}+(q-1) \frac{u_{h_{t}}^{2}}{q^{2}} \\
& =\frac{q^{2}-2 q+1+(q-1)}{q^{2}} u_{h_{t}}^{2} \\
& =\frac{q-1}{q} u_{h_{t}}^{2}
\end{aligned}
$$

for the combined uncertainty $u_{w_{t}}^{2}$ of the vertical displacements $w_{t}$. The uncertainty $u_{w_{t}}$ using equation (28) are evaluated regarding to the uncertainties of the input quantities given in Table 1 . We obtain $0.0043 \mathrm{~m}$ and $0.0059 \mathrm{~m}$ for the points 1831 and 8987 , respectively.

\subsubsection{Uncertainties obtained by the probabilistic approach}

In the probabilistic approach the random and systematic components from Table 1 are treated as having a random nature. In order to compare the performance of the MC technique to compute the uncertainties for the output quantities, the statistical moments of the PDF for every input quantities $z_{i}$ can be computed either analytically based on the statistical moments or can be estimated numerically from the generated MC samples. The analytical moments of input quantities, which are rectangular or triangular distributed are evaluated analytically according to equation (6) and (9), respectively. The expected values and variances of the normally distributed input quantities are given according to a prior knowledge or expert statements. As above mentioned, the statistical moments of all input quantities are computed also with the aid of generated $\mathrm{MC}$ samples (refer to equations (11) and (12)). The results are given by Table 2 .

The confidence intervals of every input quantity are computed only by MC simulations (refer to Section 2.2) for $\gamma=5 \%$. We identify $100000 \mathrm{MC}$ runs as necessary to obtain the estimates of the statistical moments of the PDF with at least three or four significant digits. The same results were numerically proven in Koch (2008a).

According to Section 2 we obtain the uncertainty and the confidence interval of the output quantity $y \triangleq w_{t}$ for $M=100000$ runs. The results are given in Table 3 . Comparison of uncertainties, calculated by GUM, with the result of probabilistic approach for the points 1831 and 8987, exhibits small differences of

\begin{tabular}{|c|c|c|c|c|c|c|c|}
\hline \multirow{2}{*}{$\begin{array}{l}\text { Input } \\
\text { quantity }\end{array}$} & \multirow[t]{2}{*}{ PDF } & \multicolumn{2}{|c|}{$E\left(z_{i}\right)[$ m or gon $]$} & \multicolumn{2}{|c|}{$V\left(z_{i}\right)\left[\mathrm{m}^{2}\right.$ or gon $\left.^{2}\right]$} & \multicolumn{2}{|c|}{$95 \%$ confidence interval } \\
\hline & & analytical & empirical & analytical & empirical & lower limit & upper limit \\
\hline$z_{1}$ & normal & 2.87400000 & 2.87400001 & 0.00000900 & 0.00000918 & 2.879800 & 2.868000 \\
\hline$z_{2}$ & triangular & 0.00000000 & 0.00000325 & 0.00000150 & 0.00000151 & -0.002322 & 0.0023398 \\
\hline$z_{3}$ & normal & 0.00000000 & 0.00000000 & 0.00000003 & 0.00000003 & -0.000340 & 0.0003435 \\
\hline$z_{4}$ & normal & 0.00000000 & -0.00000001 & 0.00000670 & 0.00000674 & -0.005021 & 0.0051029 \\
\hline$z_{5}$ & normal & 0.00000000 & 0.00000000 & 0.00048400 & 0.00047041 & -0.042459 & 0.0427390 \\
\hline$z_{6}$ & triangular & 14.5240000 & 14.5239607 & 0.00006667 & 0.00006613 & 14.508000 & 14.539000 \\
\hline$z_{7}$ & rectangular & 0.00000000 & -0.00000562 & 0.00083333 & 0.00083499 & -0.047558 & 0.047073 \\
\hline
\end{tabular}
the uncertainty values and confidence intervals. The confidence bounds for point 8987 is slightly asym-

Table 2: Comparison of the statistical values (expected values, variances and confidence intervals) of the analytical PDF and the empirical PDF using MC simulations for the input quantities $\mathbf{z}$.

Table 3: Uncertainties and confidence interval obtained by the MC-technique.

\begin{tabular}{lll}
\hline MC-result & Point $1831(7.28 \mathrm{~m})$ & Point $8987(21.90 \mathrm{~m})$ \\
\hline$\hat{\sigma}_{y}$ & $4.4 \mathrm{~mm}$ & $5.9 \mathrm{~mm}$ \\
$y_{\text {conf }, \mathrm{MC}}=[\underline{y}, \bar{y}]$ with $\gamma=5 \%$ & {$[-8.6 \mathrm{~mm}, 8.6 \mathrm{~mm}]$} & {$[-11.6 \mathrm{~mm}, 11.7 \mathrm{~mm}]$} \\
\hline
\end{tabular}


Table 4: Rang of values of the systematic uncertainty obtained by the fuzzy technique.

\begin{tabular}{lcc}
\hline fuzzy result (systematic component) & Point $1831(7.28 \mathrm{~m})$ & Point $8987(21.90 \mathrm{~m})$ \\
\hline$\tilde{y}_{\alpha=1, r}=\left(\tilde{y}_{\alpha=1, \max }-\tilde{y}_{\alpha=1, \min }\right) / 2$ & $0.2 \mathrm{~mm}$ & $4.8 \mathrm{~mm}$ \\
$\tilde{y}_{\alpha=0, r}=\left(\tilde{y}_{\alpha=0, \max }-\tilde{y}_{\alpha=0, \min }\right) / 2$ & $10.3 \mathrm{~mm}$ & $16.1 \mathrm{~mm}$ \\
\hline
\end{tabular}

Table 5: Random component of the uncertainty obtained by the fuzzy technique.

\begin{tabular}{lll}
\hline fuzzy result (random component) & Point $1831(7.28 \mathrm{~m})$ & Point $8987(21.90 \mathrm{~m})$ \\
\hline$\hat{\sigma}_{y}$ & $3.9 \mathrm{~mm}$ & $5.4 \mathrm{~mm}$ \\
$y_{\mathrm{conf}, \mathrm{MC}}=[\underline{y}, \bar{y}]$ with $\gamma=5 \%$ & {$[-7.6 \mathrm{~mm}, 7.6 \mathrm{~mm}]$} & {$[-10.6 \mathrm{~mm}, 10.7 \mathrm{~mm}]$} \\
\hline
\end{tabular}

Table 6: Confidence interval obtained by the fuzzy technique.

\begin{tabular}{lll}
\hline fuzzy result (confidence interval) & Point $1831(7.28 \mathrm{~m})$ & Point $8987(21.90 \mathrm{~m})$ \\
\hline$y_{\text {conf fuzzy }}=\left[\underline{y}-\tilde{y}_{\alpha=1, r} ; \bar{y}+\tilde{y}_{\alpha=1, r}\right]$ & {$[-7.8 \mathrm{~mm}, 7.8 \mathrm{~mm}]$} & {$[-15.4 \mathrm{~mm}, 15.5 \mathrm{~mm}]$} \\
$y_{\text {conf, fuzzy }}=\left[\underline{y}-\tilde{y}_{\alpha=0, r} ; \bar{y}+\tilde{y}_{\alpha=0, r}\right]$ & {$[-17.9 \mathrm{~mm}, 17.9 \mathrm{~mm}]$} & {$[-26.7 \mathrm{~mm}, 26.8 \mathrm{~mm}]$} \\
\hline
\end{tabular}

metric due to deviation of the evaluated PDF of the output quantity from the normal PDF and due to propagating of the MC samples through the nonlinear observation model. In the probabilistic approach the systematic component of the uncertainty can be reduced by repeated measurements. In order to specify this statement an illustrative example will be helpful. In general by a simple computation of the mean value the variance may be reduced if the measurement process is repeated. For more details, the reader is referred to Neumann (2009).

\subsubsection{Uncertainties obtained by the fuzzy-random approach}

In the fuzzy-random approach the treatment of the random and systematic components in the propagation process of the uncertainties is different to the probabilistic approach, see Section 3. According to Section 3 we obtain the systematic uncertainty of the output quantity $y \triangleq w_{t}$ for $\alpha=0$ and $\alpha=1$ with equations (17), (19) and (21). The results are given in Table 4

The $\alpha$-level of zero refers to the pessimistic case and the $\alpha$-level of one to the optimistic case (see Figure 3 ). Within the propagation process of the systematic component, the radius $\tilde{z}_{\alpha, r}$ of all random components $z_{i}$ from Table 1 is zero. In the presented propagation process a systematic error component cannot be reduced by repeated measurements. The rangeof-values for the systematic uncertainties cannot be decreased by mean value computation due to the mathematic rules of fuzzy theory, see Neumann (2009). The small systematic error for the Point 1831 is due to the small influence of the systematic errors of the zenith angle. For the propagation process of the random components with the methods described in Section 2, the uncertainty of the input quantities with a systematic error component are set to zero.
As a result, we obtain the uncertainty and the confidence interval of the output quantity $y \triangleq w_{t}$ for $M=100000$ runs. The results are summarized in Table 5 .

Finally, we obtain the confidence interval for the fuzzy-random approach with equation (23) for $\alpha=0$ and $\alpha=1$. The results are shown in Table 6 .

In comparison to Table 3 the random component of the uncertainties of the output quantity in the fuzzy-random approach are smaller. This is due to the reason that only the input quantities with a random uncertainty component contribute to the random uncertainty of the output quantity. These input quantities which are only affected by systematic uncertainties were not taken into account in the propagation process of random uncertainties.

In this example the reference value for the vertical height of the bridge is obtained by the mean value of the heights from each epoch. As discussed in this section in the probabilistic approach the systematic uncertainty component for the output quantity can be reduced be a mean value computation. In the fuzzy-random approach the uncertainty of a systematic component cannot be reduced by the mean value computation. This leads to a significant larger confidence interval in the fuzzy-random approach from Table 6 in comparison to Table 3. This can especially be highlighted for the pessimistic case with $\alpha=1$.

\section{3. $A$ best and worst case scenario}

The last example deals with a case study for different magnitudes for the occurring uncertainties. In order to evaluate the consequences of changing magnitudes for the uncertainties, two scenarios are computed: A best case scenario with a small uncertainty of the input quantity $z_{7}:\left(a_{+}-a_{-}\right) / 2=20$ mgon (the step width of the motor, see Table 1), and a worst case 


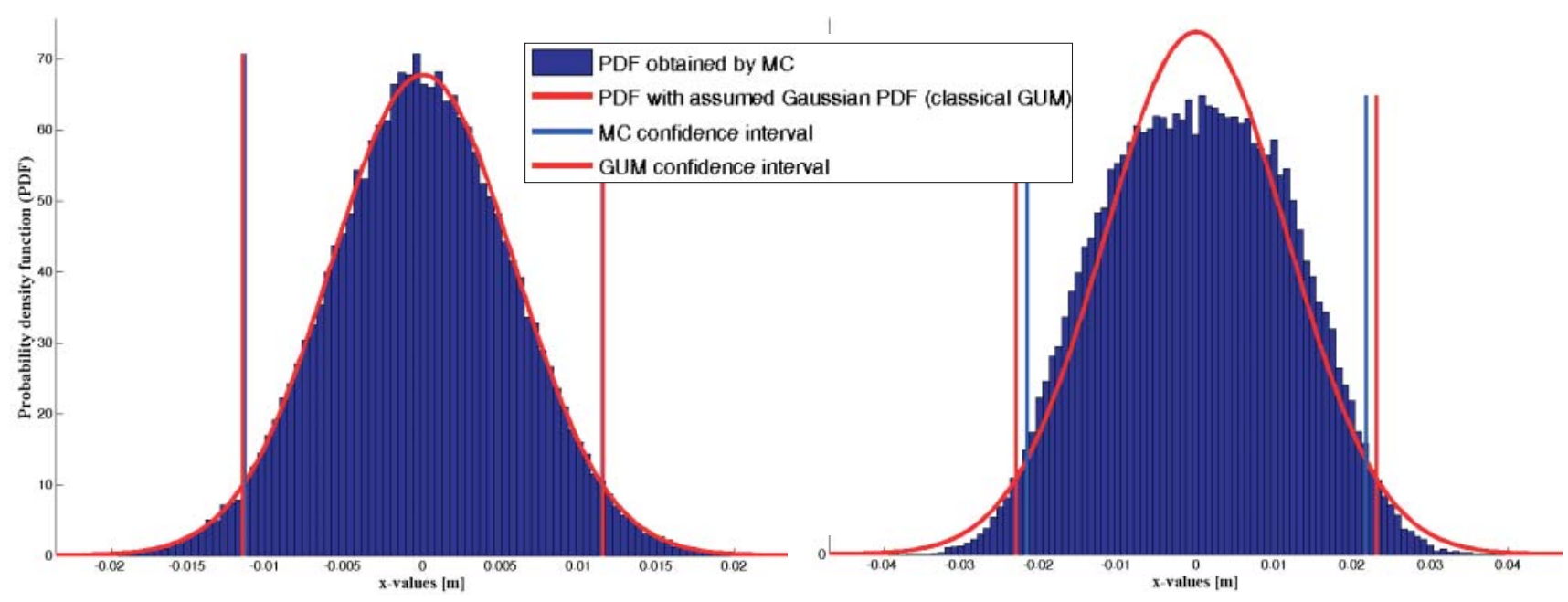

Figure 6: Comparison between the numerically computed MC-PDF and the Gaussian-PDF of the output quantity $y \triangleq w_{t}$, left the best case scenario with small uncertainties and right the worst case scenario with high uncertainties of the input quantity $z_{7}$.

scenario with high uncertainties of the input quantity $z_{7}:\left(a_{+}-a_{-}\right) / 2=50$ mgon. The assumption about the high uncertainties in the worst case scenario are realistic for very fast measurements of the laserscanner (Zoller+Fröhlich 2007).

\subsubsection{Comparing the probabilistic approach with the GUM}

Figure 6 shows the MC simulations resulting histogram for the best and the worst case scenario with corresponding theoretical normal fit and the 95\% confidence interval. In the best case scenario, the numerical results of the MC-technique and of the LOP don't show any significant differences. In the left part of Figure 6 the resulting MC PDF of the output quantity $y \triangleq w_{t}$ is compared with the GUM PDF, which is assumed to be Gaussian. The worst case scenario is introduced to find out how much the computed MC PDF and its confidence interval differ from the classical Gaussian PDF and its confidence interval. A comparison pointed out a significant difference between the uncertainty calculated by means of LOP in GUM and by means of MC techniques. The maximum difference in the worst case scenario is obtained in the confidence interval: $\max \left[\left|\underline{y}_{\mathrm{MC}}-\underline{y}_{\mathrm{GUM}}\right|,\left|\bar{y}_{\mathrm{MC}}-\bar{y}_{\mathrm{GUM}}\right|\right]=1.25 \mathrm{~mm}$. Additionally, as we can see from Figure 6, the variation in the form of both PDFs (numerical MC and Gaussian PDF) is perceivable.

This difference between the GUM and the probabilistic approach is due to the nonlinear observation model and non-Gaussian PDF of the output quantity. As mentioned before, this difference will not be significantly in the mean and the variance but rather in the estimate of the confidence interval. Overall, the probabilistic approach can be considered as an accurate and reliable approach for evaluating the uncertainty of the output quantities and its confidence interval, if the PDFs for the input quantities are known and the observation model (1), which interrelates input and output quantities, is nonlinear.

\subsubsection{Comparing the fuzzy-random approach with the GUM}

A geometrical interpretation of the systematic error of the height difference $y \triangleq w_{t}$ (output quantity) in the fuzzy-random approach is given in Figure 7. The range of values for the systematic error can be seen as a shift in the distribution of the random component. For a clear representation the distribution of the random errors of the output quantity (obtained by the MC-technique) is shown at the lower and upper bound of the systematic error. The results for the $\alpha$ levels 0 (pessimistic case) and 1 (optimistic case) are shown. When comparing the results of the fuzzy confidence interval with the results of the GUM one can clearly see, that the fuzzy confidence intervals are significantly larger. This is due to the reason that in the here presented example the systematic errors dominate the uncertainty budget. The larger the systematic errors are in comparison to the random errors, the stronger is the difference between the GUM confidence interval and the fuzzy confidence interval. This is clearly visible in Figure 7, where the left part shows the example with the best case scenario and the right part the worst case scenario. The difference of the GUM confidence interval and the fuzzy confidence interval is significantly larger in the worst case scenario.

\section{Summary and outlook}

In this paper, a measurement equation was analyzed with multidimensional input quantities and a onedimensional output quantity. The input quantities are carrier of random and systematic errors. A probabilistic and a fuzzy-random approach were introduced to handle and to propagate both types of un- 

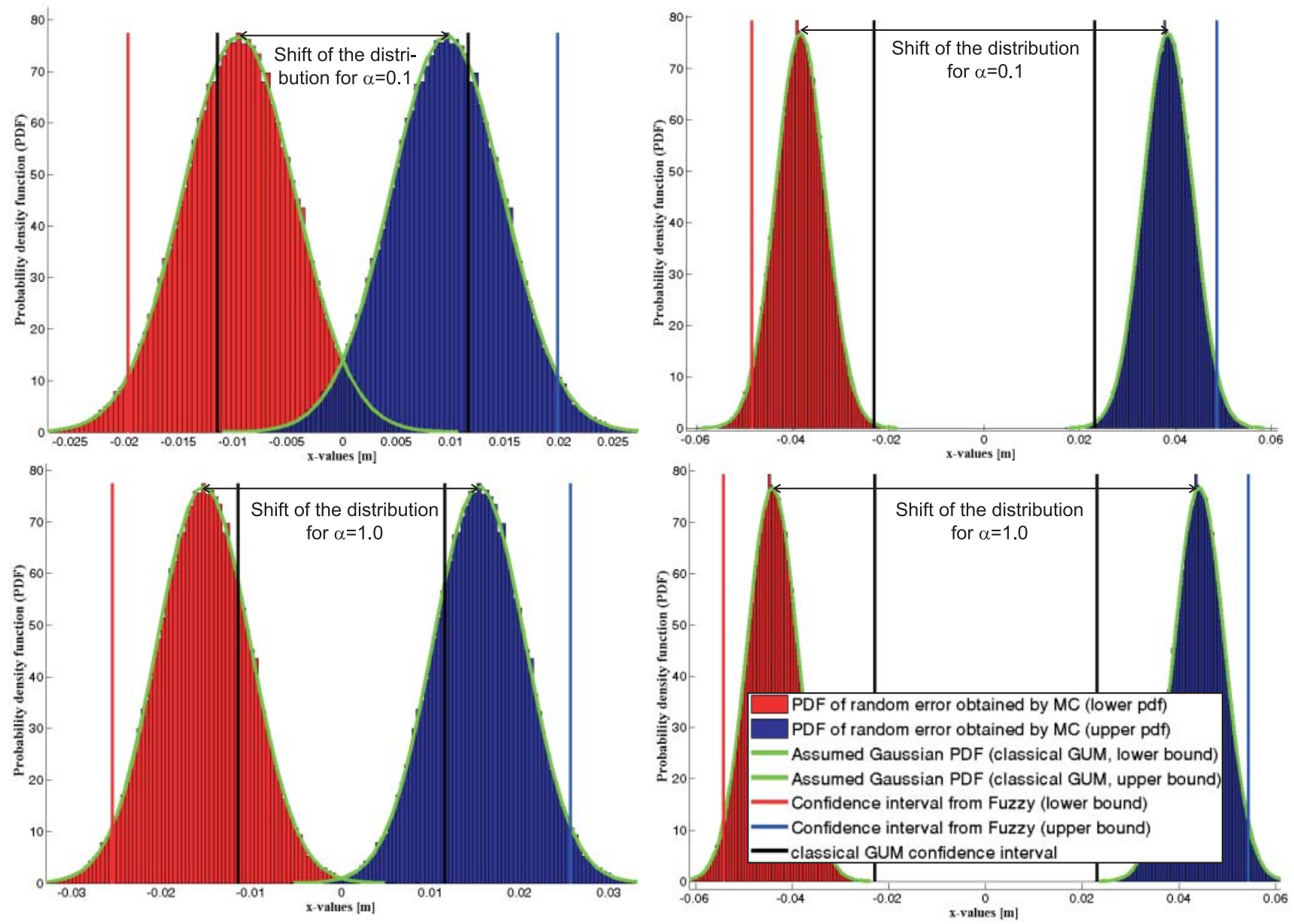

Figure 7: Comparison between the confidence intervals of the fuzzy-random approach and the GUM for the quantity $y \triangleq w_{t}$, left the best case scenario with small uncertainties and right the worst case scenario with high uncertainties of the input quantity $z_{7}$.

certainties. In the probabilistic approach both types of errors were handled as having a random nature, whereas in fuzzy-random approach the propagation process distinguishes between the random and the systematic error components. Due to the random nature in the probabilistic approach, the uncertainty of the systematic component can be reduced by averaged/repeated measurements. Therefore, it is a more optimistic representation of the uncertainties than in the fuzzy-random approach, where the systematic component of the uncertainties cannot be reduced by averaged/repeated measurements due to the mathematic rules of fuzzy theory.

Three important information can be stated from the results obtained in the paper: First, in case of strong differences from a Gaussian PDF for the random errors, the need for a more sophisticated error propagation process than in the GUM is beneficial. Second, the fuzzy-random approach allows to deal with a pessimistic and optimistic outcome for the uncertainty of the output quantity. Third, both techniques provide a rigorous consideration of all the information contained in a PDF within the propagation process of the random uncertainties to the output quantity.
Further work has to deal with an extended discussion of the presented fuzzy-random approach with input quantities having both types of uncertainties, a random and systematic component. Additionally, the bias of the output quantity resulting from the evaluation of non-linear functions has to be discussed in detail, especially in the fuzzy-random approach. Furthermore, extensions of probabilistic and fuzzyrandom approaches to handle with multidimensional correlated uncertainties in the input as well as in the output quantities have to be taken into consideration. Especially, the construction of multidimensional MC- and fuzzy confidence regions needs further studies.

\section{Acknowledgments}

The presented paper shows in parts results and new ideas developed during the research project $\mathrm{KU}$ 1250/4-2 "Geodätische Deformationsanalysen unter Berücksichtigung von Beobachtungsimpräzision und Objektunschärfe", which is funded by the German Research Foundation (DFG). This is gratefully acknowledged by the authors. 


\section{References}

Alkhatib, H., On Monte Carlo methods with applications to the current satellite gravity missions, $\mathrm{PhD}$ thesis, Institute for Geodesy and Geoinformation of University of Bonn, 2007.

Bandemer, H., Mathematics of Uncertainty - Ideas, Methods, Application Problems, Springer, Berlin, Heidelberg, New York, 2006.

Buckland, S. T., MC Confidence Intervals, Biometrica 40 (1984), 811-817.

Dubois, D. J. and Prade, H. M., Fuzzy Sets and Systems: theory and applications, Academic Press, London, New York, 1980.

Fellin, W., Lessmann, H., Oberguggenberger, M., and Vieider, R., editors, Analysing Uncertainty in Civil Engineering, Springer, Berlin, Heidelberg, New York, 2005.

Ferson, S., Ginzburg, L., Kreinovich, V., Nguyen, H., and Starks, S., Uncertainty in risk analysis: towards a general second-order approach combining interval, probabilistic, and fuzzy techniques, in: Proceedings of the 2002 IEEE International Conference on Fuzzy Systems, 2002.

Gentle, J. E., Random Number Generation and Monte Carlo Methods, Wiley, Springer, 2nd edition, 2003.

Grabe, M., Measurement Uncertainties in Science and Technology, Springer, Berlin, New York, 2005.

Hennes, M., Konkurrierende Genauigkeitsmasse: Potential und Schwächen aus der Sicht des Anwenders, AVN 4 (2007), 136-146.

ISO, Guide to the expression of uncertainty in measurements (GUM), International Organization for Standardization, Geneva, 1995.

ISO, Evaluation of measurement data - Supplement 1 to the "Guide to the expression of uncertainty in measurement" - Propagation of distributions using a Monte Carlo method, Joint Committee for Guides in Metrology, Bureau International des Poids et Mesures, Geneva, 2007.

Koch, K. R., Parameter Estimation and Hypothesis Testing in Linear Models, Springer, Berlin, Heidelberg, New York, 2nd edition, 1999.

Koch, K. R., Introduction to Bayesian Statistics, Springer, Berlin, Heidelberg, New York, 2nd edition, 2007.

Koch, K. R., Evaluation of uncertainties in measurements by Monte Carlo simulations with an application for laserscanning, J. Applied Geodesy 2 (2008a), 67-77.

Koch, K. R., Determining uncertainties of correlated measurements by Monte Carlo simulations applied to laserscanning, J. Applied Geodesy 2 (2008b), 139-147.

Kreinovich, V., Why Intervals? Why Fuzzy Numbers? Towards a new Justification, in: Proceedings of the 2007 IEEE Symposium on Foundations of Computational Intelligence (FOCI), 2007.

Kreinovich, V., Starks, S. A., Araiza, R., Xiang, G., Velasco, A. A., Averill, M. G., and Keller, G. R., Towards Combining Probabilistic, Interval, Fuzzy Uncertainty, and Constraints: An Example Using the Inverse Problem in Geophysics, in: Proceedings of the 12th GAMM - IMACS International Symposium on Scientific Computing, Computer Arithmetic and Validated Numerics (SCAN), 2006.

Kutterer, H., Zum Umgang mit Ungewissheit in der Geodäsie Bausteine für eine neue Fehlertheorie, volume 553, Deutsche Geodätische Kommission, Reihe C, München, 2002.

Kwakernaak, H., Fuzzy random variables - I: definitions and theorems, Informations Sciences 15 (1978), 1-29.

Kwakernaak, H., Fuzzy random variables - II: algorithms and examples for the discrete case, Informations Sciences 17 (1979), 253278.

Mauris, G., Lasserre, V., and Foulloy, L., A fuzzy approach for the expression of uncertainty in measurement, Measurement 29:3 (2001), 165-177.

McNish, A. G., The speed of light, IRE Transaction on Instrumentation 1:11 (1962), 138-148.
Möller, B. and Beer, M., Fuzzy Randomness: Uncertainty in Civil Engineering and Computational Mechanics, Springer, Berlin, New York, 2004.

Neumann, I., Modeling of an extended uncertainty budget in parameter estimation and hypothesis testing, treated exemplarily with the geodetic deformation analysis (in German), $\mathrm{PhD}$ thesis, Geodetic Institute, Leibniz University of Hannover, 2009, to appear.

Neumann, I. and Kutterer, H., Geodetic Deformation Analysis with respect to Observation Imprecision, in: Proceedings of the XXIII International FIG Congress, Munich, Germany, 2006.

Neumann, I. and Kutterer, H., The probability of type I and type II errors in imprecise hypothesis testing with an application to geodetic deformation analysis, International Journal of Reliability and Safety, 2009, to appear.

Nguyen, H. T. and Kreinovich, V., Nested Intervals and Sets: Concepts, Relations to Fuzzy Sets, and Applications, in: Kearfott, R. B. and Kreinovich, V., editors, Applications of Interval Computations, Kluwer Verlag, Dordrecht, 245-290, 1996.

Reshetyuk, Y., Investigation and calibration of pulsed time-of-flight terrestrial laser scanners, Licentiate thesis in Geodesy, Sweden, 2006.

Schulz, T. and Ingensand, H., Influencing Variables, Precision and Accuracy of Terrestrial Laser Scanners, in: Intergeo East, Bratislava, Slovakia, 2004.

Siebert, B. R. L. and Sommer, K.-D., Weiterentwicklung des GUM und Monte-Carlo-Techniken, Technisches Messen 71 (2004), 6780 .

Stigler, S. M., Statistics and the Question of Standards, Journal of Research of the National Institute of Standards and Technology 101:6 (1996), 779-789.

Strübing, T., Realization of a sensor integrated measuring system for the acquisition of deformation processes of structures, (in German), Geodetic Institute, Leibniz University of Hannover, 2007.

Terán, P., Probabilistic foundations for measurement modelling with fuzzy random variables, Fuzzy Sets and Systems 158:9 (2007), 973-986.

Viertl, R., Statistical Methods for Non-Precise DATA, CRC Press, New York, London, 1996.

Youden, W. J., Enduring values, Technometrics 14:1 (1972), 1-11.

Zadeh, L., Fuzzy sets, Information Control 8 (1965), 338-353.

Zoller+Fröhlich, Technical datasheet IMAGER 5006, Zoller+Fröhlich GmbH, 2007.

Received: Jan 2, 2009

Accepted: Mar 5, 2009

\section{Author information}

Hamza Alkhatib and Hansjörg Kutterer

Geodetic Institute

Leibniz University of Hannover

Nienburger Str. 1

30167 Hannover, Germany

E-mail: alkhatib@gih.uni-hannover.de

Ingo Neumann

Institute of Geodesy - Geodetic Lab

University FAF Munich

Werner Heisenberg Weg 39

85579 Neubiberg, Germany 